\title{
Datensicherheit in Datenbanksystemen
}

Hartmut Wedekind, Technische Hochschule Darmstadt

\section{Zusammenfassung}

Die Begriffe "Datenschutz", "Datensicherheit" und "Datenintegrität" werden in der Einfuhrung gegeneinander abgegrenzt. Im ersten Hauptteil werden die Sicherheitsmaßnahmen behande1t, die sich auf technische und organisatorische Belange beziehen. Die Prozesse der Identifikation und Authentifikation, die organisatorische Bildung von Schichten, Bereichen und Berechtigungsmatrizen sowie kryptographische Methoden stehen in Mittelpunkt der Betrachtungen. Der zweite Hauptteil befaßt sich mit Sicherheitsmodellen. Unter Sicherheitsmodellen verstehen wir die sprachliche Fixierung der Sicherheitsbedingungen, um diese in ein Datenverwaltungssystem einbringen zu können. Eine Datenbank beinhaltet alle gespeicherten Daten, ein Datenverwaltungssystem alle Verfahren zu ihrer Handhabung. Wir unterscheiden deskriptive (nicht prozedurale, deklarative) Sicherheitsmodelle, die für Relationale Datenbanksysteme vorgeschlagen wurden, von prozeduralen Modellen, wie sie z.B. im DBTG der CODASYL-Gruppe für hierarchische Datenbanksysteme vorgesehen sind.

\section{Einführung}

Die Begriffspaare Datenschutz und Datensicherheit auf der einen Seite und Datensicherheit und Datenintegrität auf der anderen Seite liegen so nahe beieinander, daß eine gegenseitige Abgrenzung der Begriffe vor der Behandlung von Einzelheiten erforderlich ist. Unter dem Thema "Datenschutz" soll die Frage beantwortet werden "Was und wovor ist zu schützen" (15). Man bemüht sich in dieser Disziplin um die Erarbeitung von Rechtsnormen und Organisationsvorschriften die festlegen, was aus ethischen, sozialen, wirtschaftichen oder nachrichtendienstlichen Gründen nicht jedermann zugänglich sein sol1 oder nicht in eine Daten- 
bank eingebracht werden darf. Der Datenschutz ist besonders wichtig im Hinblick auf personenbezogene Daten. Aber auch für Firmendaten (z.B. Kundenstammaten, patent-oder lizenzfähige Daten) und für Daten der öfentlichen Verwaltung ( $z$. B. Daten über Baulandp1anung) besteht ein Schutzinteresse. In Amerika ist ein Datenschutzgesetz ergangen, in Deutschland existiert ein Gesetzesentwurf der Bundesregierung.

Innerha1b der Datensicherheit interessiert man sich für die Frage "Wie ist zu schützen". Wir wollen uns in dieser Arbeit beschränken auf die Fragen der Gewährleistung einer Zugriffssicherheit, durch die unberechtigte zugriffe abgewehrt werden. Was ein unberechtigter Zugriff ist, wird durch Datenschutzvorschriften festgelegt. Wir klammern die physische Datensicherheit aus. Hierunter werden Probleme der baulichen Maßnahmen in Rechenzentren, die Schlösser- und Schlüsselverteilung, das Anbringen von Schreibringen bei Bändern, die Berücksichtigung des Feuerschutzes und die Wiederherstellung zerstörter Dateien verstanden. Die physische Datensicherheit befaßt sich mit der Sicherung vor Datenverlust.

Die Datenintegrität betrifft die Genauigkeit der Daten. Die Daten müssen Integritätsbedingungen genügen, die sich im einfachen Fall auf Datenfelder mit einer Datentypdeklaration beziehen; in komplizierten Fällen geht eine Integritätsbedingung über viele Dateien eines Datenflußplanes hinweg. Die Teile-Nr. einer Auftragsdatei müssen z.B. eine Untermenge der Teile-Nr. sein, die in der Teilestammatei aufgeführt werden. Abstimmkreise der kaufmännischen praxis sind Integritätsbedingungen, die sehr komplizierter Natur sein können. Integritätsbedingungen sind Qualitätsbedingungen der Datenbank.

Datensicherheit und Datenintegrität nennt Date (9) zu recht $z$ willingsprobleme. Datenintegrität ist die Forderung nach Fehlerlosigkeit der Datei; demgegenuber orientiert sich die Datensicherheit an Zugriff. Beide Probleme erfordern die Formulierung und das Einbringen von zusätzlichen Bedingungen. Während bei der Datensicherheit die Bedingungen aus den abstrakten Normen des Datenschutzes abgeleitet werden, berücksichtigen die Integritätsbedingungen das verwendete Datenmodell und die konkreten Spezifikationen der Miniwelt der Benutzer. Im Rahmen der Datenintegrität wird auch das Problem des möglichen Integritätsverlustes durch einen gleichzeitigen Anderungszugriff behandelt (shared access). 


\section{Sicherheit smaßnahmen}

\subsection{Identifikation und Authentifikation}

Wenn ein Benutzer zu einer Datenbank (DB) Zugang haben wi11, so muß er sich zuerst identifizieren, d.h., er muß dem System sagen, wer er ist. Diese Identifikation muß auf Richtigkeit überprüft sein, sie muß authentifiziert werden. Ein weitverbreitetes Mittel zur Identifikation aber auch zur Authentifikation ist bei DB Systemen das Kennwort (password). Jeder Benutzer bekommt ein Kennwort. Die Kennworte werden in einer Kennworttabelle vom DB-System verwaltet. Die Tabelle kann noch weitere Personenstammdaten wie Personal-Nummer, Name und Datum der Kennworterteilung enthalten. In der Tabelle kann ferner vermerkt werden, welche Terminale benutzt werden dürfen. Damit kann überprüft werden, ob der Benutzer an einem vorher identifizierten Terminal uberhaupt arbeiten darf. Der Prozeß der Identifikation wird bei manchen Terminalen heute dadurch vereinfacht und auch sicherer gemacht, daß maschinenlesbare Ausweiskarten verlangt werden. Mit der Personenidentifikation geht im System die Terminalidentifikation einher. Dem System wird so bekannt, wo die Terminalsitzung stattfindet. Wenn man davon ausgehen kann, daß die Kennworte und ihre Abspeicherung geheim bleiben, so ist der Identifikationsprozeß auch gleichzeitig ein Authentifikationsprozeß. Damit die Annahme realistischer wird, können besondere Methoden für die Kennwortvergabe eingeführt werden. Sehr sicher, aber auch aufwendig, ist ein Kennwort, das nur einmal benutzt werden kann (one time password). Petersen und Turn (20) führen aus, daß diese Art der Kennwortvergabe auch nicht vor solchen Eindringlingen schützt, die sich mit einem Terminal in den Terminalbetrieb zwischen dem berechtigten Benutzer und dem System einschalten (infiltration between the lines). Die Kennworttabelle mu auf jeden Fall geheim bleiben, wenn sie nicht in einer Geheimschrift verschlüsselt wird. Eine weitere Möglichkeit, Identifikation und Authentifikation zusammenfallen zu lassen, wäre die Überprüfung von Finderabdrücken, die Analyse der maschinellen Stimme oder die Unterschriftskontrolle. Für auch wirtschaftlich vertretbare Verfahren sind getrennte Identifikations- und Authentifikationsprozesse notwendig. Bei einer Authentifikation sind Informationen auszunutzen, die nur der Person bekannt sind, die sich in der Identifikationsprozedur als solche ausgegeben hat. Man kann zum Beispie1 ein weiteres Kennwort angeben müsen. Allein schon durch ein "Uber-die-Schulter-gucken" kann auch dieses Kennwort al1gemeir bekannt werden. Zweckmässiger ist es desha1b, daß das system dem Benutzer, der 
einen Zugang wünscht, eine Frage ste11t, die nur dieser beantworten kann. Auf Vorschlag von L. Earnest empfiehlt Hoffman (16, S. 92) wie folgt vorzugehen. Beim "log-in" identifiziert der Benutzer sich; er bekommt daraufhin vom System eine Pseudozufal1szahl angeboten, die wir $x$ nennen wollen. Durch eine einfache Transformation $T$, die vom Benutzer im Kopf durchzufuhren ist, wird eine Zahl y ermitte1t. Das Ergebnis $y=T(x)$ wird eingegeben. Das System vollzieht ebenfalls die Transformation $\mathrm{T}(\mathrm{x})$ und prüt, ob das Ergebnis tatsächlich $y$ ist. Ein potentieller Eindringling kann höchstens $x$ und $y$ sehen. Die Transformation $T$ ist für ihn kaum in Erfahrung zu bxingen, wenn die Prozedur im Rechner geschützt ist. Die Prozedur ist aber geschützt, da nur der Zugriff hat, der authentifiziert worden ist. Es kann für $\mathrm{T} z . B$. die folgende Transformation vorgeschlagen werden, die kaum von einem Dritten ermittelt werden kann:

$$
\mathrm{T}(\mathrm{x})=\left(\sum_{\mathrm{i} \text {-te }} \text { iffer } \text { von } \mathrm{x}\right)^{2}+(\text { Stunde des Tages })
$$

Es werden also die ziffern auf den ungeraden stellen summiert. Die Summe wird quadriert und zur stunde des Tages addiert.

Die dargestellte Methode zur Authentifikation ist sehr einfach und wenig aufwendig. Sie hat darüber hinaus den Vorteil, daß die Kennworttabelle jedermann bekannt sein kann, da sie zur Identifikation benötigt wird. Geheim bleiben muß lediglich $\mathrm{T}(\mathrm{x})$.

Weitere Methoden zur Authentifikation werden von Evans u.a. (10) und Purdy (21) vorgeschlagen. Beide Verfahren ähneln sich sehr stark und bauen auf Erkenntnissen auf, die innerhalb der Kryptographie (Geheimschrift oder Chiffrekunde, kryptos=geheim) entwickelt wurden. Wegen der großen Bedeutung der Kryptographie für die Sicherheit von DBSystemen werden wir in einem gesonderten Abschnitt auf diese Verfahren eingehen. Auf die erwähnten Verfahren von Evans und Purdy, die auf der Methode der "Ein-Weg Chiffre" (one way cipher) von Wilkes (27) aufbauen, sol1 hier in diesem Rahmen nicht eingegangen werden.

\subsection{Schichtungen, Bereichsbildungen und Berechtigungstabellen}

Es gibt drei einfache strukturen, um ein Sicherheitssystem zu organi-sieren. Es handelt sich um die Schichtung (stratification), die Bereichsbildung oder Sektionierung (compartmentalization) und die Anordnung der Zugriffsberechtigung in einer Berechtigungstabelle (authorization table).

Bei der Schichtung werden die Benutzer im Hinblick auf die Zugriffsberechtigung im Sinne einer Hierarchie in Gruppen eingeteilt. Die Schichten der Daten oder die Benutzergruppen erha1ten $z . B$. von oben 
nach unten die folgenden Bezeichnungen:

$\begin{aligned} \text { streng geheim } & 1 . \text { Schicht } \\ \text { geheim } & 2 . \text { Schicht } \\ \text { streng vertraulich } & \text { 3. Schicht } \\ \text { vertraulich } & \text { 4. Schicht } \\ \text { nicht klassifiziert } & 5 . \text { Schicht }\end{aligned}$

Eine Person, die z.B. Zugriff zu streng geheimen Daten hat, um diese zu sehen, zu löschen oder zu ändern, hat auch Zugriff zu Daten in darunter liegenden Schichten. Kann eine Person hingegen nur zu streng vertraulichen Daten zugreifen, so bleiben die Schichten "streng geheim" und geheim" für sie unzugänglich. Allgemein gilt: Eine Person darf nur zu den Daten der Schicht, für die sie klassifiziert wurde, und $z u$ Daten in darunter liegenden Schichten zugreifen. Die Schichtung von Personen und Daten aus Gründen der Sicherheit stammt aus dem militärischen Bereich. In zivilen Sicherheitssystemen ist diese Sicherheitsorganisation ungebräuch1ich. Aber auch im militärischen Bereich kombiniert man häufig die Schichtung mit der Bereichsbildung, die im Englischen "compartmentalization" heißt. Bei der Bereichsbildung werden die Daten in disjunktive Teilmengen zerlegt. Eine Teilmenge oder ein Bereich (Sektion) wird einer Person oder auch einer Personengruppe zugeordnet. Daten dürfen nur genau einmal in einem "Bereich" vorhanden sein. Das Sicherheitssystem muß gewährleisten, daß zwischen den Bereichen Sperren liegen, die nicht durchbrochen werden können. Martin (19,S.151) sieht die Bereichsbildung als eine vertikale Aufteilung der Daten. Die Schichtung wird von ihm auch horizontale Aufteilung genannt.

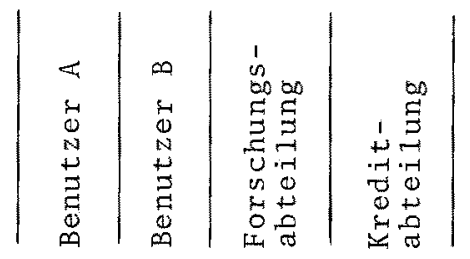

In einem vertikalen Bereich sind bei personengruppen auch horizontale Schichten denkbar. Dìese Form wird häufig bei militärischen Sicherheitssystemen vorgefunden. Auch hier möchte man, daß eine 
Person nux Zugang zu den Daten hat, die von ihr auch wixklich gebraucht werden. Friedman $(14,5.269)$ nennt die Bereichsbildung eine Umsetzung des militärischen Postulats des "Need-To-Know". Jeder sol1 nur das wissen, was er wirklich benötigt. Eine sehr bekannte Anwendung der Bereichsbildung ist die speichergeschüzte Aufteilung des Zentralspeichers für Einzelprogramme. Das Betriebssystem gewährleistet beim Multiprogrammingbetrieb, daß in einem Programm nicht der Speicherbereich eines anderen Programms adressiert werden kann. Unterstützt wird das Betriebssystem dabei häufig hardwaremäßig durch Begrenzungsregister (base limit register). Ein Register dieser Art nimmt eine Basisadresse und die Bereichslänge auf. Durch Vergleich der Programmadressen mit dem Registerinhalt kann eine Bereichsüberschreitung entdeckt werden. Die dritte Form der einfachen Strukturen für ein Sicherheitssystem ist die Berechtigungstabelle. Die Tabelle enthält das Kennwort, die Personal-Nr. und ein n-bit-langes Feld für die Berechtigung. Ist das $i-t e$ Bit eine 1 , so ist ein Zugriff zum Sicherungsobjekt $\mathrm{D}_{i}$ erlaubt, bei 0 wird der Zugriff verwehrt. Die Tabelle wird auch häufig Benutzerprofil (user security profile) genannt. Der Nachteil ist, daß die binäre Rege1 "entweder Zugriff oder kein Zugriff" gilt. Bei Datenbanksystemen wird diese Tabelle häufig als Matrix ausgebildet, wobei die zeilen die Benutzer und die Spalten die Sicherungsobjekte darstellen. Eine Berechtigungsmatrix soll an einem Beispie1 erklärt werden, daß in einer ähnlichen Form auch bei Conway u.a. (8, S.212) zu finden ist. Wir gehen aus von der Relation PERSONAL (PNR, LSTG, GHT, LMB, VST), die außer der Personal-Nr (PNR) die sehr sensitiven personaldaten Leistung (LSTG), Gehalt (GHT), letzter medizinischer Befund (LMB) und Vorstrafen (VST) enthä1t. Die bereits behandelte Kennworttabelle dient zur Zeilenidentifikation.

\begin{tabular}{|c|c|c|c|c|c|c|c|}
\hline PNR & Kennwort & PNR & LSTG & GHT & LMB & VST & Bemerkung \\
\hline A & $13 \mathrm{C} 151$ & $\mathrm{R}$ & $\mathrm{R}, \mathrm{W}$ & $\mathrm{R}, \mathrm{W}$ & $\mathrm{R}$ & $\mathrm{R}, \mathrm{W}$ & Personalchef \\
\hline B & $74 \quad 0028$ & $R, W$ & $\mathrm{R}$ & $\mathrm{R}$ & $\mathrm{N}$ & $N$ & Organis.-Chef \\
\hline $\mathrm{C}$ & $43 \mathrm{~F} 974$ & $\mathrm{R}$ & $N$ & $\bar{N}$ & $\mathrm{~N}$ & $\mathrm{~N}$ & Programmierer \\
\hline $\mathrm{D}$ & $14 \quad Z \quad 234$ & $R$ & $\mathrm{~N}$ & $\bar{N}$ & $\mathrm{R}, \mathrm{W}$ & $\mathrm{N}$ & Mediziner \\
\hline $\mathrm{F}$ & 28 R 862 & $\mathrm{~N}$ & $\mathrm{R}$ & $\mathrm{R}$ & $\mathrm{R}$ & $R$ & Statistiker \\
\hline
\end{tabular}

$R=$ Lesen erlaubt, $W=$ Verändern erlaubt

$N=$ Weder Lesen noch Verändern erlaubt 
Damit in einem System die Berechtigungsmatrizen für Mengen von entities nicht zu speicheraufwendig werden, wird empfohlen, Zonen und Kategorien zu bilden ( 19. S.6). Eine Zone ist dabei die Zusammenfassung mindestens zweier Mengen von Sicherungsgegenständen. Aus den Mengen Verkaufsteil, Einkaufsteil und Fertigungsteil wird. die Zone Teil. Eine Kategorie ist die Zusammenfassung mehrerer Attribute. Aus Kunden-Name, Wohnort und Umsatz kann die Kategorie "Kundeninformation" entstehen. Eine weitere Reduktion des Speicheraufwandes ist die Bildung von Benutzergruppen. Alle Mitglieder einer Benutzergruppe haben genau gleiche Zugriffsrechte. Zwecks leichter sprachlicher Unterscheidung wird eine Benutzergruppe, die aus sicherungstechnischen Grüden gebildet wird, von Friedman (14.S.269)"C1ique" genannt. Zonen, Kategorien und Cliquen sind drei sehr einprägsame Begriffe.

Gegenüber den Schichtungen und Bereichsbildungen läßt die Berechtigungsmatrix schon die Darstellung von wesentlich subtileren Sicherheitsbedingungen $z u$. Die Sicherheitsbedingungen dürfen jedoch nicht wertabhängig sein. Eine tabellarische Darstellung in der Form des Datenschemas "Matrix" ist dann nur noch sehr schwer möglich. Es muß zu einer sprachlichen Formulierung der Sicherheitsbedingungen übergegangen werden. Eine Bedingung ist dann wertabhängig, wenn Attributausprägungen zu ihrer Formulierung benötigt werden.

Man kann wertabhängige Sicherheitsbedingungen von beliebiger Komplexität angeben. Die Vorschriften des Datenschutzes verlangen häufig die Einhaltung nur einfacher wertabhängiger Bedingungen, wie z.B. die Personalsatzbedingung. Wertunabhängige Bedingungen können zur übersetzungszeit, wertabhängige Bedingungen erst zur Ausführungszeit überprüft werden. Wertabhängige Bedingungen sind sehr zeitaufwendig.

\subsection{Umgehung der Sicherheitsvorkehrungen}

In diesem Abschnitt werden Methoden zur Umgehung der Sicherheitsmaßnahmen beschrieben. Gleichzeitig wird die Frage behandelt, welche Sicherheitsmaßnahmen gebraucht werden, um mit Vorsatz arbeitende Eindringlinge abzuwehren. Bei den Methoden der Eindringlinge handelt es sich um "Schurkereien", die den "naiven" und "rechtschaffenen" Benutzer sehr esotorisch anmuten. Für viele Angriffe der Eindringlinge ist die Verschlüsselung der Datenbank im Sinne der Kryptographie eine wirkungsvolle Gegenmaßnahme. Die Attacken und Verteidigungsmaßnahmen auf ein DV-System sind in vorzüglicher weise in dem viel beachteten *) d.h. jeder darf nur seinen eigenen Persona1stamnsatz Iesen. 
Aufsatz von Peterson und Turn (20) dargeste11t.

Die Ziele eines vorsätzlichen Eindringens in ein DB-System können sein:

1) Gewinnung von Information, 2) Herausfinden, welches Informationsinteresse ein Benutzer hat, 3) Andern und Zerstören von Information, 4) Kostenlose Nutzung von Resourcen des Systems oder Nutzung von Systemresourcen auf Kosten eines anderen.

Von Peterson und Turn werden die Methoden (20) zum vorsätzlichen Eindringen in das System in zwei Kategorien eingeteilt. Es wird von passiver Infiltration gesprochen, wenn der Eindringling sich auf irgendeine Weise in das DV-System einschaltet, um zu wissen, was vor sich geht. Eine aktive Infiltration liegt dann vor, wenn der Eindringling entweder Systemressourcen nutzen will oder gezielt Informationen gewinnen, ändern oder zerstören wil1. Die Methoden der passiven Infiltration sind das Anzapfen von Ubertragungsleitungen (wiretapping) vom System zum Terminal und das Anbringen von Sonden (electromagnetic pickups) in CPU und diversen Speichern. Die Ubertragungsleitungen gelten als der Teil des Gesamtsystems, der am leichtesten verletzbar ist $(20,5.291)$. Nach Peterson und Turn setzt man sich gegen diese beiden aufgefühten Angriffe am besten durch eine Verschlüsselung in eine Geheimschrift zur Wehr. Dem Eindringling wird dann aufgebüdet, die Chiffre zu "knacken". Wenn der Aufwand (work factor) zum Brechen der Chiffre größer ist als der Wert der gewonnenen Information, Iohnen sich diese Angriffe nicht. Diese Aussage ist sehr abstrakt, da zwar der Aufwand zur Codebrechung nicht aber der wert der Information für einen Eindringling abgeschätzt werden kann. Im Hinblick auf die aktive Infiltration können die folgenden Methoden aufgezäht werden:

1) "Masquerading". Der Eindringling hat sich z.B. über ein Anzapfen der Leitung das Kennwort eines Benutzers besorgt und "maskiert" sich nun mit diesem. Durch Verschlüsseln kann verhindert werden, daß der Eindringling durch Anzapfen das Kennwort erfährt. Das Verschlüsseln und Entschlusseln muß selbstverständlich am Terminal stattfinden.

2) "Browsing" (Schnüfeln). Der Eindringling ist ein rechtmäßiger Benutzer, der den Identifikations- und Authentifikationsprozeß erfolgreich passiert. Er versucht jedoch Daten zu lesen oder zu verändern, zu denen er nicht zugreifen darf. Eine gut funktionierende zugriffskontrolle ist die beste Abwehr gegen diese Art der Infiltration. 
3) In die Obertragungsleitung zwischen Benutzer und System wird vom Eindringling ein eigenes Terminal eingebracht. Während der rechtmäßige Benutzer am Terminal sitzt, kann sich folgendes ereignen:

a) Der Eindringling löscht das "sign-off" Kommando und fährt fort, im Namen des Benutzers sein Terminal zu bedienen. Dieser glaubt, daß die Terminalsitzung beendet sei.

b) Während das Terminal des rechtmäßigen Benutzers inaktiv ist, schaltet sich der Eindringling ein, um mit der Datenbank $z u$ arbeiten ("between the lines").

c) Der Eindringling sucht sich die spezielle Information aus dem Verkehr zwischen dem rechtmäßigen Benutzer und dem system aus, verändert diese und läßt die modifizierte, fehlerhafte Information zum Terminal des Benutzers übertragen. ("piggyback entry").

Die Verschlüsselung ist für diese drei Fälle die beste Gegenwehr.

4) Diebstahl eines auswechselbaren Datenträgers. Neben der physischen Absicherung durch speziel1 verschließbare Räume ist auch hier die Verschlüselung zu empfehlen.

5) Die Eindringlinge sind Systemprogrammierer mit Detailkenntnissen auf dem Gebiet des Speicherschutzes, des Programierens im privilegierten Modus und des Betriebssystems. Eindringlinge dieses Typs sind naturgemäß die gefährlichsten. Sie können absichtlich undichte Stellen in Systemprogramme einbauen (trap doors) oder sich von Zeit zur Zeit den Zentralspeicher herausdrucken lassen. Die Systeme sind heute so kompliziert, daß nur ein Team von Eindringlingen erfolgreich arbeiten kann, was einen gewissen Schutz darstel1t, da ein ganzes Team sich nur in seltenen Fällen auf eine "Schurkerei" dieser Art einläßt. Durch das Protokollieren gewisser Operationen, wie zum Beispiel das Herausdrucken des Zentralspeichers, können nachträglich unzulässige Eingriffe bekannt werden. Peterson und Turn nennen diese Maßnahmen "threat monitoring"; sie messen ihnen eine große Bedeutung bei. Besonders schwer zu erkennen sind Angriffe, die durch Software-Modifikationen, z.B. durch Änderungen von Ubersetzern, Vorübersetzern, Texteditoren etc. zustande kommen. Da fast alle Programme durch andere Programme verarbeitet werden, stellt Bayer $(1,5.78)$ zu recht den Grundsatz auf : Kein Programm ist sicherer als diejenigen Programme, durch die es bearbeitet wird". 
Systemprogramierer mit Detailkenntnissen können auch die Methode des "eingepflanzten Satzes" benutzen, um die Chiffre schneller zu brechen. Der Eindringling bringt Klartextfragmente in die Datei und spürt dann die Chiffre zur Codebrechung auf. Insbesondere Bayer ( 1 ) hat auf diesen Vorgang aufmerksam gemacht.

\subsection{Kryptographische Methoden}

Kryptographie ist die Lehre von der Erzeugung eines Geheimtextes aus einem ursprünglichen Text und von der Wiedergewinnung eines ursprünglichen Textes aus einem Geheimtext. Der erste Vorgang heißt Chiffrieren; sein Umkehrung wird Dechiffrieren genannt. Andere Bezeichnungen für "ursprünglichen Text" und "Geheimtext" sind "Klartext" und "Kryptogramn" oder "Chiffre". Eine Chiffre ist eine unverständiche Folge von Schriftzeichen. Die Sicherheit eines Chiffrierverfahrens beurteilt man nach dem Widerstand oder Aufwand (work factor) für den unberufenen Eindringling. Im einem kryptographischen Code, der keine Chiffre ist, können Teile der Schriftzeichenfolge von einem Dritten zwar verstanden, aber nicht richtig gedeutet werden. Es werden Wörter und ganze Satzteile ziemlich willkürich ausgetauscht. Wie dieser Austausch durchzuführen ist, wird in einem wörterbuch, das Codebuch geannt wird, festgehalten. Bei kryptographischen Codes soll in der Regel auch eine Datenkompression erzielt werden. Wegen des Speicheraufwandes, der durch das Codebuch verursacht wird, kommen kryptographische Codes für DB-Systeme nicht in Betracht. Wir benötigen algorithmische Chiffrier- und Dechiffrierverfahren und keine tabellarischen. Es können drei Arten von algorithmischen Verfahren unterschieden werden:

a) Ersetzungsverfahren (substitution methods), b) Versetzungsverfahren (transposition methods) c) Block-Chiffrierverfahren (block cipher methods)

Die Kryptographie hat eine lange Geschichte. Liebende und Diebe haben ihre Verbindungen immer so gut es eben ging verheimlicht, bemerkt Feistel $(13,5.21)$, um in scherzhafter weise auf die vorwissenschaftliche Kryptographie einzugehen. Erst etwa Mitte des vergangenen Jahrhunderts wurde die Kryptographie langsam zu einer Wissenschaft. Der geheime Nachrichtenaustausch bleibt jedoch bis tief in dieses Jahrhundert hinein auf Bleistift und Papier beschränkt. Durch den Computer hat die Kryptographie dann einen neuen, kaum erwarteten Aufschwung genommen. Alle historischen Anmerkungen, die wir im Verlauf der Darstellung machen werden, sind aus dem berühmten Buch von Kahn "The Codebreakers" (18).

a) Ersetzungsverfahren

Bei diesem kryptographischen Verfahren wird ein Zeichen 
des Klartextes durch ein Zeichen aus dem Alphabet der Chiffre ersetzt. Im Gegensatz zum einfacheren Versetzungsverfahren bleibt die Identität eines Klartextzeichens nicht erhalten. Man kann das Ersetzen über eine Tabelle oder algorithmisch, d.h. in diesem zusammenhang algebraisch durchführen. Innerhalb der Datenbank-Kryptographie haben die additiven Substitutionsverfahren wegen ihrer hohen Leistungsfähigkeit bei großen Datenmengen eine besondere Bedeutung bekommen. Von den additiven Verfahren wollen wir hier die Verfahren "Addition modulo q" oder die Vignère-Vemam-Chiffren hervorheben.

Im frühen 16. Jahrhundert hat der Benediktinermönch Trithemus das uberhaupt erste gedruckte Buch über Kryptographie veröffent1icht. Trithemus beschreibt in diesem Buch eine quadratische Matrix mit Buchstaben als Elemente, deren Zeilen von oben nach unten jeweils um eine Position versetzt werden. Er benutzte diese Matrix als Schlüse1 zum Chiffrieren in einem additiven Substitutionsverfahren. Im späten 16. Jahrhundert wurde diese Idee von Blaise de Vignere wieder aufgegriffen und verbessert. Historisch nicht ganz korrekt wird das im folgenden dargeste11te Verfahren Vignere-Verfahren genannt.

\section{$\underline{\text { K1artext }}$}

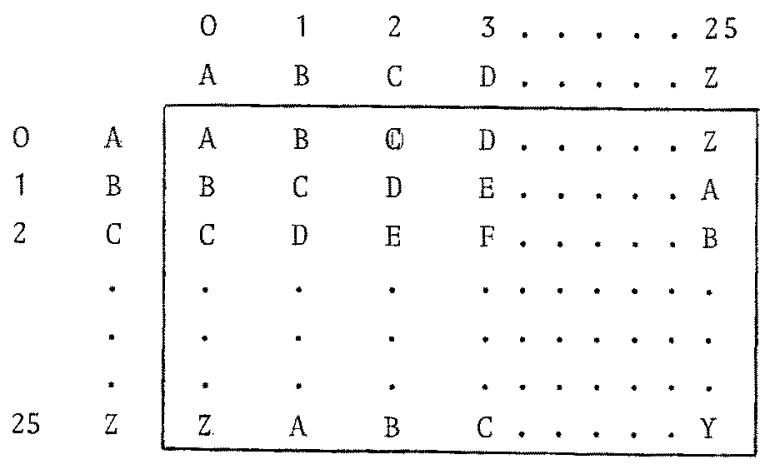

Schlüsselmatrix für die Vignère-Chiffre

Die Spaltenbezeichnungen ( $A, B, C$, etc) gelten für den Klartext. Die Zeilenbezeichnungen werden für den Schlüssel benötigt. Das Chiffrezeichen wird im Schnittpunkt zwischen Zeile und Spalte gefunden. Wenn einem D im Klartext ein B im Schlüssel gegenübersteht, so finden wir als Chiffrezeichen E. Beim Dechiffrieren geht man umgekehrt vor. 
Wir wollen "klassische" Chiffrierung mit der Vignère-Methode an einem Beispiel demonstrieren:

Gegeben sei der Klartext :"KEIN VERRATER" und der Schlüsse1 "KAISERBALL"

K1artext : K E I N V E R R A E T E R Schlüsse1: KA IS E R B A L L K A I Chiffre: $\quad U E Q F Z V S R L P D E Z$

Wenn wir den Buchstaben $A$ bis $Z$ die Zahlen $O$ bis 25 zuordnen, wie das in der Abb. geschehen ist, so kann man den Chiffrierungsprozess als Addition modulo 26 auffassen. Für das obige Beispiel finden wir dann, ohne die Matrix benutzen zu müssen:

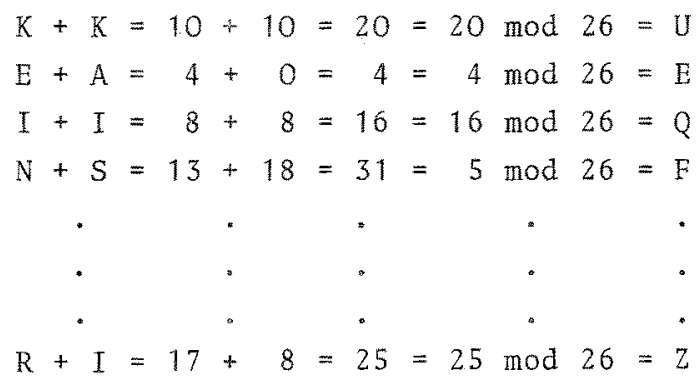

Im vergangenen Jahrhundert nannte man die Vignère-Chiffre "le chiffre indechiffrable". Tuckerman (23), kann zeigen, daß das Brechen dieser Chiffre mit Computermethoden kein großes Problem ist, wenn genügend Geheimtext vorhanden ist. Nicht zu brechen ist ein Geheimtext, wenn der Schlüssel nur einmal benutzt und durch einen zufallszahlengenerator erzeugt wird, der keine periodischen Pseudozufallszahlen produziert. Je länger der Schlüssel, desto schwieriger wird es, die Chiffre zu brechen.

Der amerikanische Nachrichteningenieur Gilbert Vernam hat 1917 die Vignère-Chiffre zum ersten Mal auf einen digitalisierten Datenstrom angewendet. Vernam sah sich vor die Aufgabe gestellt, ein Alphabet zu chiffrieren, das aus $2^{5}=32$ zeichen für Fernschreiber bestand, und in einem Digitalcode dargeboten wurde. Das Ergebnis seiner Untersuchungen war eine Addition modulo 2.

Beispie1

Chiffrieren:

Klartext: $\quad 011001: 111100$ '

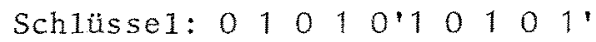
Chiffre: $\quad 000011.010011$
Dechiffrieren:

Chiffre: $\begin{array}{lllllllllll}0 & 0 & 0 & 1 & 1 & 0 & 1 & 0 & 0 & 1\end{array}$

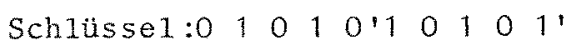
Klartext: $\quad 0 \quad 1 \quad 0 \quad 0 \quad 11111100$ r 
Die Addition modulo 2 und die logische Operation "Exklusives ODER" sind identisch. Mit dem "Exklusiven ODER" wird auch wieder dechiffriert. Das "Exklusive ODER" hat eine eindeutige Inverse. Die Vernam-Chiffrierung spielt in der Datenbank-Kryptographie eine besondere Rolle. Sie ist eine spezielle Chiffre nach dem "Addition modulo q" Verfahren, das von Tuckermann Vignère-Vernam-System oder abgekürzt V-V-System genannt wird.

b) Versetzungsverfahren

Bei der Anwendung von Versetzungsverfahren bleibt die Identität des Zeichens gewahrt, es ändert sich lediglich die Position. Kinder praktizieren z.B. eines der vielen versetzungsverfahren, wenn sie ihren Namen von hinten nach vorne hinschreiben.

Eine gebräuchliche Methode für eine Versetzungstransformation ist das Aufteilen des Klartextes in Blöcke mit einer anschlieBenden Anordnung in einer Matrix. Auf die Matrix werden einige Operationen angewendet. Der so entstandene Text wird dann wieder nach einer bestimmten Rege 1 in die 1ineare Form gebracht. Da Versetzungsverfahren a1leine angewendet $z u$ wenig Sicherheit bieten - eine Häufigkeitsanalyse der Zeichen kann schon zum Ziele fuhren - wollen wir sie nicht genauer beschreiben.

c) Block-Chiffrierverfahren

Ein Chiffrierverfahren, das $n$ Informationsbits in $n$ Chiffrebits umwande1t, wird Block-Chiffrierverfahren genannt. Die Bit-VernamTransformation ist in diesem Sinne ein Block-Chiffrierverfahren. Wenn man heute jedoch den Begriff "Block-Chiffrierverfahren" ohne weitere Zusätze benutzt, dann denkt man an ein Verfahren, das die folgenden Eigenschaften hat:

1. Ersetzen (substitution) und Versetzen (transposition) werden stufenweise hintereinander angewendet. Da das "Hintereinanderschalten" von Prozessen eine "multiplikative" Verknüpfung bedeutet, werden BlockChiffrierverfahren auch Produkt-Chiffrierverfahren genannt.

2. Im Gegensatz $z u$ den "Bit für Bit" oder "Buchstaben für Buchstaben" Ersetzungsverfahren, bei denen in der Chiffre keine Abhängigkeiten zwischen den einzelnen Bits oder Buchstaben bestehen, wird der Block aus $n$ Bits als Ganzes behandelt. Es liegt in der Chiffre eine Symbolabhängigkeit vor. Wenn ein Symbol in der Chiffre von einem anderen abhängig ist, kann durch einen Ubertragungsfehler bei einem Symbol ein ganzer Block nicht mehr dechiffrierbar sein. 
3. Die Stufe "Substitution" ist eine nichtlineare Transformation. Auf diesen Eigenschaften werden wir im folgenden noch genauer eingehen.

Block-Chiffrierverfahren für die Rechneranwendung wurden insbesondere von Feistel entwickelt (13), (12) und (11). Sie zeichnen sich durch große Sicherheit aus, d.h. der Aufwand, eine Block-Chiffre $\mathrm{zu}$ brechen, ist beträcht1ich. Block-Chiffrierverfahren wurden schon von der deutschen Armee im ersten weltkrieg unter den Namen ADFGVX benutzt (18). Zwischen den We1tkriegen wurden dann Chiffrier-Maschinen entwickelt, die mit einem Pseudozufallschlüssel arbeiteten. Erst die Rechneranwendungen haben Mitte der 60-iger Jahre wieder das Interesse für Block-Chiffrierverfahren belebt $(13,5.99)$. Wir werden die BlockChiffrierverfahren hier so darstellen, als würden die Transformationen von Gexäten besorgt.

Wir beginnen mit der Beschreibung der nichtlinearen Substitution.

\begin{tabular}{|l|l|}
\hline Eingang & Ausgang \\
\hline 000 & 011 \\
\hline 001 & 111 \\
\hline 010 & 000 \\
\hline 011 & 110 \\
\hline 100 & 010 \\
\hline 101 & 100 \\
\hline 110 & 101 \\
\hline 110 & 003 \\
\hline
\end{tabular}

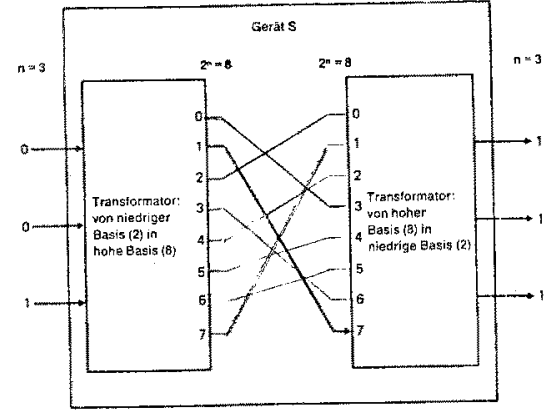

Nichtlineare Substitution nach Feistel (13, S.25)

Es wird angenomen, daß der Klartext in Blöcken zu $n=3$ Bits eingegegeben wird. Es werden nicht wie beim Vernam-Verfahren eine Null oder eine Eins $z u$ den Eingabeziffern addiert, sondern man substituiert einen ganzen Eingabeziffernblock durch einen beliebigen Ausgabeziffernblock. Das Substitutionsgerät (Gerät $S$ ) besteht im wesentlichen aus zwei Basistransformatoren. Der Eingabetransformator nimmt einen Block in der Darstellung zur Basis 2 auf und verwandelt ihn in eine oktalzah1. Der Ausgabetransformator geht umgekehrt vor. Für die n Eingabebits gibt es $2^{\text {n }}$ Substitutionszeichen, die auf nichtiineare Weise durch Basistransformation gefunden werden. Die Basistransformation ist auch als ein gutes Verfahren zur Erzeugung von hash-Adressen bekannt. Bevor eine Substitution im umgekehrten Sinne stattfindet, wird 
eine Versetzung vorgenommen, die man sich durch eine einfache verdrahtung realisiert yorstellen kann. Eine mögliche Verdrahtung wird in der Abbildung gezeigt. Insgesamt gibt es $2^{n} !=8 !=40320$ solcher verdrahtungen (Hardware) oder Tabellen (Software). Für $n=3$ oder 4 ist ein Substitutionsgerät mit einex beliebigen Verdrahtungsmöglichkeit noch zu realisieren. Durch Eingeben gewisser "Tricknachrichten" kann der Eindringling jedoch die Chiffre brechen $(13,5.26)$. Das ist nicht mehr der Fall, wenn z.B. $n=128$ gewählt wird. Mit 128 Ein- und Ausgängen müßte der Eindringling $2^{128}>10^{38}$ verschiedene Blöcke eingeben, um die Arbeitsweise des Gerätes $z u$ erforschen. Das ist nicht durchführbar. Diesem Vorteil steht der entscheidende Nachteil gegenüber, daß das Verfahren für große $n, z . B$. für $n=128$, technisch nicht realisiert werden kann. Dies ist die Ursache, die dazu führte, daß man mehrere kleinere Substitutionsgeräte, $z \cdot B$. mit $n=3$, in einer Stufe parallel anordnete. Da eine Substitutionsstufe mit mehreren S-Geräten noch sehr 1eicht überwunden werden kann, wurde ein Versetzungsgerät nachgeschaltet. Da in einem Versetzungsgerät eine. Permutation von Symbolen

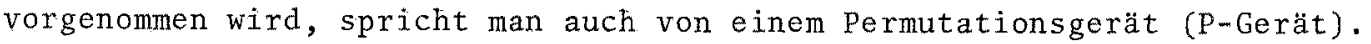
Kaum zu brechen ist eine Chiffre dann, wenn mehrere P-Geräte und $\mathrm{S}-\mathrm{Ge}-$ räter hintereinander angeordnet werden. Das "Durcheinanderwirbeln" der Bits durch eine komplizierte Transformation $\mathrm{H}$ ist so groß, daß eine Inversion für jemanden, der nicht eingeweiht ist, kaum noch nachvol1zogen werden kann. Die Abbildung zeigt, wie aus einer einzigen 1 durch mehrmaliges nichtiineares Substituieren und Permutieren (Versetzen) eine "Lawine" von Einsen entstehen kann. Bei der Dechiffrierung werden die Stufen in umgekehrter Richtung durchlaufen. Um das Block-Chiffrierverfahren für den Gegner noch schwieriger $z u$ machen, können für die SGeräte jeweils andere Schlüssel vorgegeben werden, so daß wir zwischen den Geräten $s_{1}, s_{2}, \ldots \ldots \ldots s_{20}$ unterscheiden können.

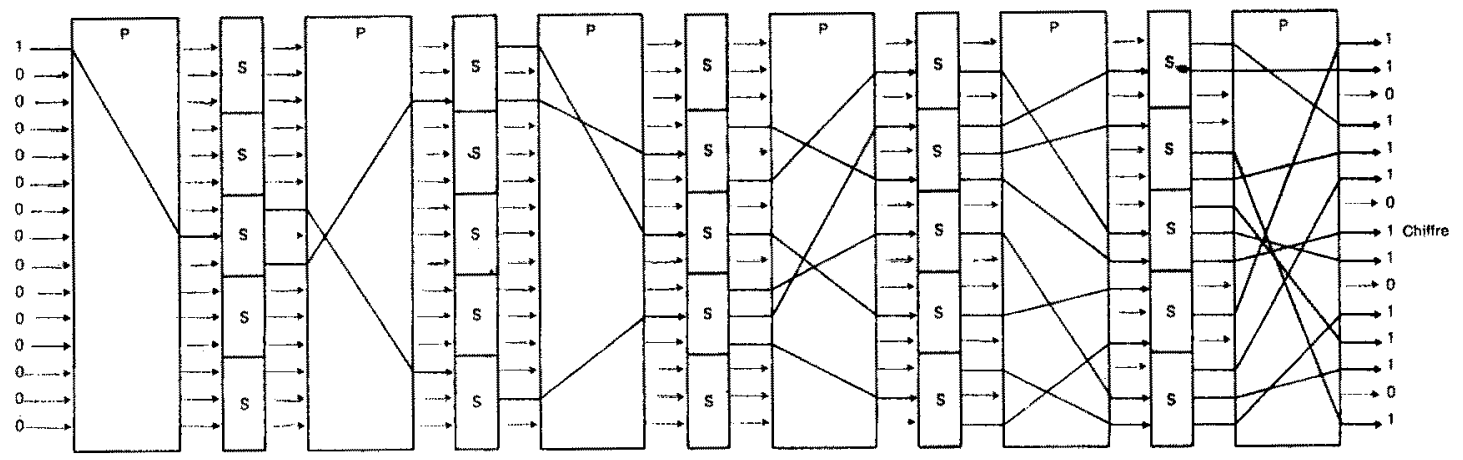

Block-Chiffrieren nach Feiste1 (13, S.100) 


\section{Sicherheitsmode11e in Datenbanksystemen}

\subsection{Deskriptive Mode11e}

Relationale DB-Systeme unterscheiden sich von anderen DB-Systemen dadurch, daß mehrere Entwurfsebenen deutlich erkennbar sind. Es können drei Ebenen exwähnt werden, die in einem relationalen DBSystem mindestens vorhanden sein müssen:

1. Die logische Ebene, 2. Die Ebene der Zugriffspfade, 3. Die Ebene der physischen Abspeicherung auf den Geräten.

In DB-Systemen, die auf dem Relationenmodell der Daten basieren, wird davon ausgegangen, daß Sicherheitsbedingungen zur Miniwe1t des Benutzers gerhören und daß ihre Formalisierung deskriptiv auf der logischen Ebene erfolgen muß. Sicherheitsbedingungen und auch Integritätsbedingungen werden prinzipiel1 genauso behandelt wie Anfragen (queries) der Benutzer. Sicherheits- und Integritätsbedingungen können sehr komplex sein. Es werden keine Sicherheitsorganisationen in Form von Schichtungen, Bereichsbildungen oder Berechtigungstabellen vorausgesetzt.

Gegeben sei eine relationale Datenbank mit $n$ Relationen $R_{1}, R_{2}, \ldots, R_{n}$. Mit Hilfe einer Datenmanipulationssprache (DML) mit dem Relationenmode11 als Grundlage (etwa ALPHA ( 7 ), SQUARE (3) oder SEQUEL (4)) ist es möglich, logische Bedingungen so zu formulieren, daß das Ergebnis der Qualifikation die Beantwortung einer Anfrage ist. In ALPHA wurde die Beantwortung einer Anfrage Zieliste (target list) genannt. In Anlehnung an Chamberlin ( 5 ) und Boyce (2) wollen wir das Ergebnis der Anfrage "Sicht" (view) nennen. Eine Sicht ist eine Relation, die nicht abspeichert, sondern nur durch logische Bedingungen definiert wird. "Views" sind virtuelle Relationen. Wenn die Basisrelationen (base relations) $R_{1}, R_{2} \ldots, R_{n}$ verändert werden, die tatsächlich zur Abspeicherung anstehen, so werden auch die abgeleiteten, virtuellen Relationen einer Anderung unterworfen. Eine "Sicht" ist im Sinne von Chamberlin ( 5 ) ein dynamisches Ergebnis einer Anfrage, in der auch built-in Funktionen wie CounT, SUM etc. benutzt werden dürfen. Der Begriff "Sicht" stammt von Boyce (2). Er wird eingeführt, um insbesondere die Sprache SEQUEL auch als Datenbeschreibungssprache (Data Description Language, DDL) herauszustellen. Im Sinne einer DDL liegt nur dann eine vollständige Sicht vor, wenn alle Beschreibungsparameter einer Basisrelation deklariert werden. Sichten im Sinne einer DDL sollen an den Basisrelationen

LAGER_GUT (NR, BEZ, MENGE, PREIS)

LIEFERUNG (NR, LNR, DATUM) 
veranschaulicht werden. Es bedeuten: NR = Nummer des Lagergutes, LNR = Lieferanten-Nummer, BEZ = Bezeichnung.

Die formale Beschreibung von LAGER-GUT lautet:

DEFINE LAGER_GUT TABLE AS:

NR $(\underline{S C O P E}=$ POSINT,,$\underline{R E P R}=\mathrm{DEC}(6))$

BEZ $($ SCOPE $=A L P H A$, DOMAIN $=$ NAME , REPR $=$ CHAR $(*)$

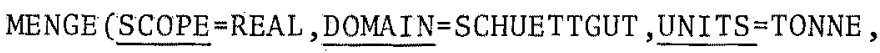
REPR=FLOAT DEC $(15,4)$

PREIS $($ SCOPE $=$ REAL, DOMAIN $=G E L D$, UNITS $=D M$ PRO TONNE, $\underline{\mathrm{REPR}}=$ FLOAT DEC $(8,2)$

$\mathrm{KEY}=\mathrm{NR}$,

ORDER=ASCENDING TNR

INDEX, BEZ

DEFINE LIEFERUNG TABLE AS:

NR LIKE LAGER GUT.NR

LNR LIKE NR EXCEPT (REPR=DEC (8))

DATUM ( $\underline{\text { SCOPE }}=\mathrm{POSINT}, \underline{\mathrm{REPR}}=\mathrm{DEC}(3))$

KEY NR, LNR

ORDER $=$ DESCENDING NR, ASCENDING LNR

Eine Tabelle wird zunächst durch ihren Tabellennamen, die Namen der Spalten und - wenn notwendig - durch die Ordnung der Zeilen beschrieben. Eine Spalte (Attribut) kann kenntlich gemacht werden durch einen Namen, einen Wertebereich (SCOPE) z.B. positive ganze zah1 (POSINT), eine Vergleichbarkeit (comparability DOMAIN), die aussagt, ob zwei Werte vergleichbar sind, eine Maßeinheit (UNITS) z.B. Tonne und eine Darstellung (REPR, representation). Die Begriffe SCOPE, UNITS und REPR erklären sich selbst. Zu bemerken ist nur, daß gewisse standardausprägungen wie POSINT, REAL etc. für SCOPE und etwa FIXED BINARY, DECIMAL etc. für REPR bereitgestel1t werden sollten. Was die Vergleichbarkeit anbetrifft, so sind zwei Werte nur dann vergleichbar, wenn sie aus Spalten stammen, für die der Parameter DOMAIN gleich ist. "Schütgüter" können nur mit "Schüttgütern" und "Geld" kann nur mit "Geld" verglichen werden. Insbesondere dann, wenn mit zwei Relationen ein Verbund gebildet werden soll, spielt die Vergleichbarkeit eine große Rolle. Zur Veranschaulichung des Begriffes "Sicht" im Hinblick auf Sicherheitsbedingungen wird im folgenden ein Beispiel in der Sprache SEQUEL dargeste11t:

Aus der Basisrelation "LAGER_GUT" sol1 die Sicht "VERKAUFS GUT" entwickelt werden. Ein Verkaufsgut unterscheidet sich dabei von einem Lagergut dadurch, daß das Schüttgut in säcke abgepackt und nach stücken 
gezäh1t wird. Die Sicht "VERKAUFS GUT" enthä1t das Lagergut in einem verkaufsfähigen, abgepackten Zustand. Ein Stück, d.h. ein Sack sol1 1/100 Tonnen wiegen.

DEFINE VERKAUFS_GUT TABLE AS:

LIKE LAGER GUT EXCEPT (MENGE. DOMAIN=SAECKE, MENGE. UNITS=STUECK, PREIS. UNITS=DM PRO STUECK)

Uber die folgende Deklaration wird dem System die Umrechnung Tonnen in Stuck mitgeteilt.

DEFINE CONVERT (TONNE TO STUECK):

$1 / 100$ TONNE

CONVERT kann aIs Umrechnungsroutine aufgefaßt werden. Um die Umrechnung selber braucht sich der Benutzer der Sicht "VERKAUFS_GUT" nicht zu kümmern. Um die abgeleitete Sicht "VERKAUFS GUT" zu einer Sicherheitsbedingung $z u$ vervoliständigen, muß geklärt werden, was dem Benutzer einer Sicht alles erlaubt ist. Wir stellen uns dabei vor, daß wir der Eigentümer der Basisrelation LAGER_GUT sind und volle Verfügungsgewalt über diese Relation haben. Der Begriff "Eigentümer" wird in diesem Sinne definiert. Chamberlin u.a. (5) schlagen nun die folgenden Verfügungsrechte vor:

1) GRANT (Gewähren): Hiermit wïrd verfügt, daß der Benutzer der abgeleiteten sicht diese Sicht jedem beliebigen anderen Benutzer zeigen darf. Anders ausgedrlickt: Die Weitervergabe der Leseerlaubnis wird gewährt.

2) REVOKE (Widerrufen): Die Verfügung über die Sicht wird widerrufen.

3) DESTROY (Zerstören): Hiermit wird die Erlaubnis zum Zerstören der Sicht erteilt.

4) INSERT (Einfügen): Es wird zugestanden, Tupeln in die virtuelle Relation (Sicht) einzufügen.

5) DELETE (Löschen): Es dürfen Tupeln gelöscht werden.

6) UPDATE (Modifizieren): Attribute dürfen verändert werden.

Eine Sicht und Verfügungsrechte machen eine Sicherheitsbedingung aus. Dem Benutzer mit der $\mathrm{Nr}$. X sollen die folgenden Rechte zugestanden werden:

GRANT VERKAUFS GUT TO BENUTZER. BNR $=$ ' $\mathrm{X}$ '

$$
\begin{aligned}
& \left(\text { GRANT }={ }^{\prime} \text { NO', REVOKE }=' \text { 'NO', DESTROY }='\right. \text { 'NO' } \\
& \underline{\text { INSERT }}={ }^{\prime} \text { NO }^{\prime}, \underline{\text { DELETE }}={ }^{\prime} \text { NO', PREIS. UPDATE }={ }^{\prime} \mathrm{YES}^{\prime} \text { ) }
\end{aligned}
$$


Wir wollen die folgenden Merkmale für Sichten und Sicherheitsbedingungen herausstellen:

1. Verschiedene Sichten können hierarchisch wie "Generationen von Geschlechtern" aufgebaut werden. Wir unterscheiden "Vater-Sichten" von "Sohn-Sichten". An der Wurzel des Baumes steht der "Ur-Vater", die Basisrelation, die dem Eigentümer gehört. Alle Anderungen in einer "Vater-Sicht" werden in den diversen 'Sohn-Sichten' berücksichtigt. Es wurde bereits der Grundsatz aufgeste11t, Anderungen im allgemeinen nur beim "Ur-Vater" vorzunehmen.

2. Die Verfügungen oder Berechtigungen, die einer "Sohn-Sicht" zustehen, müssen immer im Umfang kleiner oder gleich sein dem Umfang, den die "Vater-Sicht" hat. Die "Sohn-Sicht" muß aus der "Vater-Sicht" produziert werden können. (Nemo plus juris transferre potest, quam ipse habet).

3. Beim Widerrufen einer Sicht werden die zugehörigen "Sohn-Sichten" zerstört.

Aus der Sicht VERKAUFS_GUT soll in einem weiteren Beispiel für den Benutzer $Y$ eine Sicht entwickelt werden, damit er nur die Felder NR und BEZ lesen kann.

Wir schreiben in der Sprache SEQUEL:

DEFINE LISTE FÜR Y'1 TABLE AS:

SELECT NR, BEZ

FROM VERKAUFS_GUT

Es folgt dann:

GRANT LISTE FÜR Y 1 TO BENUTZER. BNR $=$ ' $\mathrm{Y}$ '

(GRANT $={ }^{\prime}{ }^{\prime}{ }^{\prime}$, REVOKE $^{\prime}{ }^{\prime}{ }^{\prime} \mathrm{NO}^{\prime}, \underline{\text { DESTROY }}={ }^{\prime}$ 'NO'

$\underline{\text { INSERT }}=$ 'NO', DELETE 'NO', UPDATE $=$ 'NO')

\subsection{Prozedura1 Modelle}

Bevor wir zu einer kurzen Darstellung von prozeduralen Modellen übergehen, wie sie im DBTG-Report ( 6 ) und bei Hoffman (16) ausfuhrlicher $z u$ finden sind, verweisen wir hier auf die Sicherheitsmerkmale des Systems IMS (siehe (26)). Alle Zugriffe zu einer IMS-Datenbank gehen über einen zugeordneten PCB (Program Communication Block), der ein Subschema beschreibt. Damit wird von der Architektur her eine besondere Sicherheitsstufe vorgesehen. Es kann von einem Programm nur zu Daten zugegriffen werden, die durch ein $P C B$ sensitiv gemacht wurden. Das Programm darf zweitens nur solche Operationen ausführen, die im Felde PROC-OPTIONS des PCB definiert wurden. Die kleinste Sicherungseinheit 
ist ein Segment. Eine Berechtigungsmatrix kann mit Hilfe des IMS über PROC-OPTIONS implementiert werden. Weitere Sicherungsmöglichkeiten bietet das IMS an, wenn der Datenkommunikationsteil installiert wird. Es kann dann z.B. spezifiziert werden, daß Programme nur bei Angabe von Kennworten aufgerufen werden dürfen und daß auch ein Kennwort erforderlich ist, um an Terminalen gewisse Kommandos benutzen zu dürfen.

Für Datenbanksysteme sind das system DBTG (6) und das von Hoffman entwickelte "Formulary Model"(16) zwei wichtige Repräsentanten für Sicherheitsmodelle, in denen die Sicherheitsbedingungen von einer Zentralstelle direkt in Sicherheitsprozeduren (von Hoffman "Formularies" genannt) umgesetzt werden müssen. Dabei steht dem Programmierer eine Trägersprache, etwa PL/1 oder COBOL zur Verfügung. Wir wollen uns in unserer Darstellung auf die wichtigsten Merkmale im system DBTG beschränken.

In der Sprache des DBTG ist die kleinste, nicht weiter auflösbare Dateneinheit ein "data-item". Ein Name und eine Ausprägung machen ein "data-item" aus. Mehrere "data-items" mit einem gemeinsamen Namen sind ein "data-aggregate". Mehrere "data-items" oder "aggregates" bilden einen "data-record", der wie alle Einheiten bezeichnet sein muß. Die wesentliche hierarchische Struktur im Datenmodell des DBTG ist der "set". In einem set gibt es solche Sätze, die "owner" (Vatersatz) und solche, die "membex" (Sohnsatz) heißen. Eine Ausprägung eines "set" (set occurrence) besteht aus genau einem "owner record" mit keinem, einem oder mehreren"member records". Ein "member record" kann ohne einen "owner record" nicht existieren (Integritätsbedingung). In einem "set" ist ein "record" Typ entweder "owner" oder "member" (aber nicht beides). Ein "member record" kann "member record" in mehreren "sets" sein. Damit sind dann Netzwerkstrukturen darstellbar. Ein "set" ist streng zu unterscheiden vom mathematischen Begriff "set" (Menge). Eine weitere Organisationseinheit ist eine "area". Der gesamte Speicherbereich einer DBTG-Datenbank wird in eine Anzah1 von bezeichneten "areas" zerlegt. Ein "schema" enthält die gesamte formalisierte Beschreibung einer Datenbank. Ein "sub-schema" ist grob gesprochen eine vom Benutzer ausgewählte Untermenge eines "schema". Ein Programm kann nur zu solchen Daten zugreifen, die in einem "sub-schema" definiert sind. Wie im rMS, in dem das "sub-schema" durch einen PCB festgelegt wird, ist auch im DBTG durch das "sub-schema" eine gewisse Sicherheit in einer ersten Stufe gewährleistet. Die zweite Stufe wird im folgenden beschrieben. Das "sub-schema" kann als ein sehr starres und eingeengtes 
Sicht -Konzept aufgefaßt werden.

Das System DBTG unterstützt Sicherheitsprozeduren im Hinblick auf alle angeführten Organisationseinheiten vom "data-item" bis zum "schema". Um nur das Wesentliche hier darzustellen, werden wir uns auf das "record-Niveau" beschränken.

Vom DBTG werden zwei Klauseln bereitgestellt: $\mathrm{Zu}$ einem PRIVACY LOCK im "schema" und zum anderen ein PRIVACY KEY im Programm des Benutzers. Mit Hilfe der Datenbeschreibungssprache (DDL) für das "schema" kann eì "Schloß" (PRIVACY LOCK) vor die Daten "gehängt" werden, das mit Hilfe eines "Schlüssels" (PRIVACY KEY), formuliert in der Datenmanipulationssprache (DML), geöffnet werden kann. Im einfachsten Fall ist der Schlüssel ein Kennwort, in komplizierteren Fällen wird eine Prozedur aufgerufen. Wir stellen zunächst den Fall dar, daß der Schlüssel als Kennwort aufzufassen ist. In der Sprache COBOL als Trägersprache soll im folgenden das Programm des Benutzers aufgefaßt sein. Die Sicherheitsbedingung möge lauten: Nur wer über das Kennwort 'KATSERBALL' verfügt, darf einen beliebigen Satz mit dem Namen PERSONAL 1öschen:

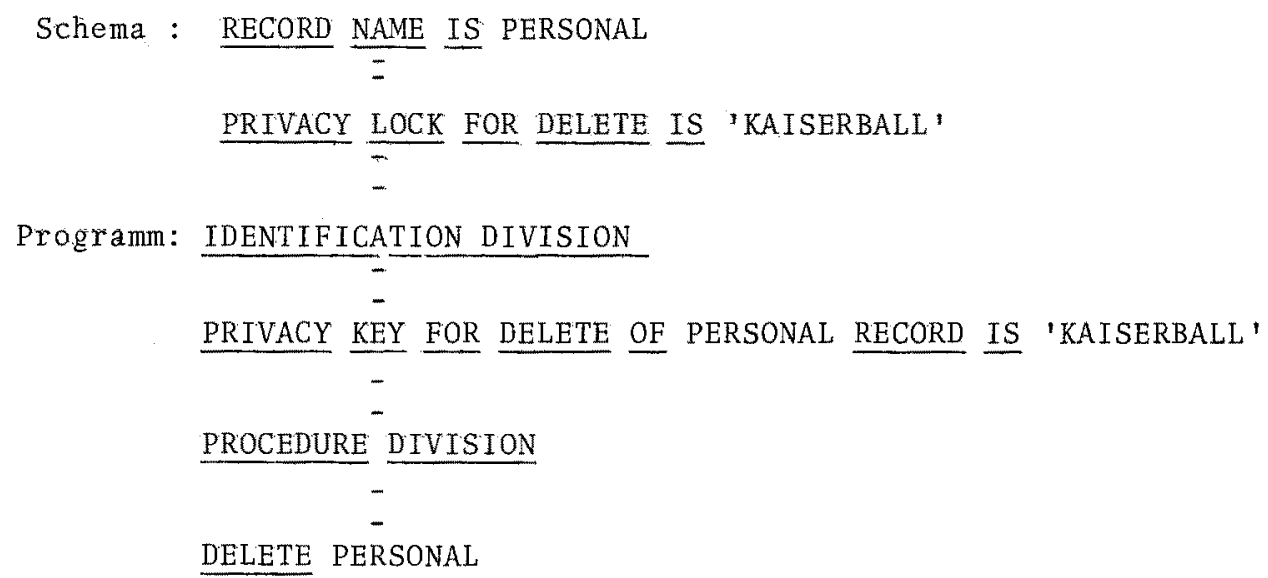

Das DBTG sorgt dafür, daß die Zeichenkette 'KAISERBALL' in der Klausel PRIVACY LOCK mit der Zeichenkette 'KAISERBALL' in der Klausel PRIVACY KEY verglichen wird. Bei Gleichheit der Zeichenketten wir die Operation DELETE PERSONAL im Hinblick auf einen beliebigen Satz PERSONAL erlaubt. Bei Ungleichheit der Zeichenkette wird die Operation unterdrückt und ein Fehlerstatus-Anzeiger gesetzt.

Wir kommen nun zu dem komplizierteren Fall, der dann vorliegt, wenn die Ausfuhrung einer Operation nicht von einem Kennwort sondern von Bedin- 
gungen abhängt. Es soll von dem folgenden Beispie1 ausgegangen werden: Ein Personalleiter darf Sätze mit dem Namen PERSONAL dann 1öschen; wenn 1. der Inhalt des Feldes Gehalt kleiner als 10.000 ist oder wenn 2. der Inhalt des Feldes Abteilungs-Nummer gleich 15 ist.

Die erste Bedingung sei in der Prozedur mit dem Namen GAMMA und die zweite in der prozedur mit dem Namen DELTA realisiert. In den folgenden Anweisungen wird angenommen, daß der personalleiter nur aufgrund der 1. Bedingung einen Zugriff wünscht.

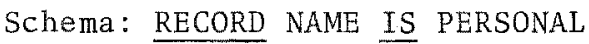
-

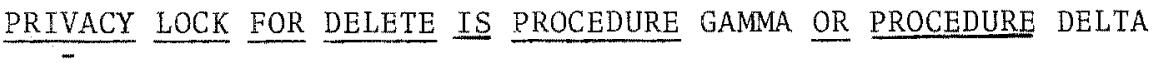

Programm: IDENTIFICATION DIVISION

$=$

PRIVACY KEY FOR DELETE OF PERSONAL RECORD IS PROCEDURE GAMMA

PROCEDURE DIVISION

-

DELETE PERSONAL

Mehrere PRIVACY LOCKS können durch ein OR zusammen in einer Anweisung definiert werden. In einer Prozedur wird die Zugriffsberechtigung geprüft. Die Prozedur übergibt den Parameter 'Ja' oder 'Nein' dem DBTG. Die Prozeduren selber werden im DBTG nicht weiter spezifiziert. Hoffman (16) jedoch gibt die Struktur einiger Sicherheitsprozeduren an. Man kann davon ausgehen, daß für jede Sicht mit den dazugehörigen Aktionen im Sinne des vorherigen Abschnitts eine Prozedur zur Verfügung gestellt werden muß. Bei einem komplizierten Sicherheitssystem mit vielen verwickelten Sicherheitsbedingungen wird der Installation eine beacht1iche Arbeit aufgebürdet, wenn die prozedurale Lösung gewäh1t wird. 


\section{Literaturverzeichnis}

1) Bayer, R, und Metzger, J. U.: On the Encipherment of Search Trees and Random Process Files, Institut für Informatik, TU München, März 1975.

2) Boyce, R. F. und ChamberIin, D. D.: Using a structured English query language as a data definition facility, IBM Research Report, $\mathrm{Rj} 1318$, San José, Dec. 10, 1973.

3) Boyce, R. F. u. a.: Specifying Queries as a Relational Expression: SQUARE, in: Proc. ACM SIGPLANSIGIR Interface Meetings Gaitherburg, Maryland, Nov. 4-6, 1973.

4) Chamberlin, D. D. U. a.: A Structured English Query Language, in: Proc. ACM SIGFIDET Workshop on Data Description Access and Contro1, Ann Arbor, Mich., May 1-3, 1974.

5) Chamberlin, D. D., Gray, J. M und Traiger, I. L.: Views, Authorization and Locking in a Relational Data Base System, IBM Research Report, Rj 1486, Sam José, Dec. 19, 1974.

6) CODASYL DATA BASE TASK GROUP (DBTG) REPORT, April 1971, erhält1ich bei IFTP Administrative Data Processing Group, 40 Paulus Potterstraat, Amsterdam.

7) Codd, E. F.: A data base sublanguage founded on the relational calculas, in: 1971 ACM SIGFIDET Workshop on Data Description, Access and Control, San Diego, Nov. 11, 1971, S. 35-68.

8) Conway, R. W., Maxwe11, W. L. and Morgan, H. L.: On the Implementation of Security Measures in Information Systems, in: Com. ACM, Vo1. 15 (1972), No. 4, S. 211-220.

9) Date, C. J.: An Introduction to Data Base System, Addison Wesley, Reading (Mass.), 1975.

10) Evans, A. und Kantrowitz, W.: A User Authentication Scheme not requiring Secrecy in the Computer, in: Com. ACM, Vo1. 17 (1974), No. 8, S. 437-442.

11) Feistel, H., Notz, W. A. und Smith, J. L.: Cryptographic techniques for machine to machine data communication, IBM Research Report, RC 3663, Yorktown Heights, Dec. 27, 1971.

12) Feiste1, H.: Cryptographic coding for data-bank privacy, IBM Research Report, RC 2827 , Yorktown Heights, 1970.

13) Feistel, H.: Chiffriermethoden und Datenschutz, in: TBM Nachrichten, Teil 1, 24. Jg. (1974), Heft 219, S. 21-26. Teil 2, 24. Jg. (1974), Heft 220, S. 99-102. Öbersetzung aus dem Englischen: Feiste1, H., Cryptography and computer privacy, in: Scientific American, Vol. 228 (1973), No. 5, S. 15-23. 
14) Friedmann, T. D.: The authorization problem in shared files, in: IBM Systems Journa1, Vol. 9 (1970), No. 4, S. 258-280.

15) Hentsche1, B., Gliss, H., Bayer, R. und Dierstein, B.: Datenschutzfibel, Verlag J. P. Bachem, Köln 1974.

16) Hoffman, L. J.: Computer and Privacy, A Survey, in: Computing Surveys, Vol. 1 (1959), No. 2, S. 85-103.

17) IBM-Broschüre: The Consideration of Physical Security in a Computer Environment, Oktober 1972, Fr. Nr. 6520-2700-0.

18) Kahn, D.: The Codebrakers, McMillan, New York, 1967.

19) Martin, J.: Security, Accuracy and Privacy, Prentice Ha11, Englewood Cliffs, 1973.

20) Petersen, H. E. und Turn, R.: System Implication of Information Privacy, in: AFIPS Conf. Proc., Vol. 30 (1967), SJCC, Thompson Book, New York, S. 291-300.

21) Purdy, G. B.: A High Security Log-in Procedure, in: Com. ACM, Vol. $17(1974)$, No. 8, S. 442-445.

22) Stonebraker, M. und Wong, E.: Access Control in a Relational Data Base Management System by Query Modification. University of California (Berkeley) Research Report ERL-M438, 14 May, 1974 .

23) Tuckerman, B.: A Study of the Vigenère-Vernam Single and Multiple Loop Enciphering Systems, IBM Research Report, RC 2879, Yorktown Heights, May 14, 1970.

24) Turn, R.: Privacy Transformation for Databank Systems, Rand Corporation, Forschungsbericht für die National Science Foundation, AD-761563, March 1973, veröffentlicht auch in: AFTPS Conf. Proc. Vol $42(1973)$, S. 589-601.

25) Turn, R.: Privacy and Security in Personal Information Databank Systems, (Prepared for the National Science Foundation), Rand Corporation, R-1044-NSF, March 1974.

26) Wedekind, H. und Härder, Th.: Datenbanksysteme II, Bibliographisches Institut, Mannheim, 1975. (noch unveröffenticht)

27) Wilkes, M. V.: Time-Sharing Betriebe bej digitalen Rechenanlagen (Ubersetzung aus dem Englischen), Carl-Hanser Verlag, München, 1970.

28) Scherf, J.A.: Computer and Data Security, A Comprehensive Annotated Bibliography, MIT Project MAC, January 1974 